\title{
A NOTE ON SLIT MAPPINGS
}

\section{BY DOV AHARONOV}

Communicated by Prof. Wolfgang H. Fuchs, February 24, 1969

1. Introduction. Recently the unitary properties of Grunsky's matrix have been studied by several authors. Milin [5] was apparently the first to observe these properties, and Pederson [6], unaware of Milin's work, rediscovered them independently later.

Let $f(z)=z+\sum_{k=2}^{\infty} a_{k} z^{k}$ be a regular univalent function in the unit circle. The function

$$
\log \frac{f(z)-f(\zeta)}{z-\zeta}=\sum_{n, k=0}^{\infty} d_{n k} z^{n} \zeta^{k}
$$

is then regular in $|z|<1,|\zeta|<1$.

Grunsky's matrix $B=\left(b_{n k}\right), b_{n k}=(n k)^{1 / 2} d_{n k}, n, k=1,2, \cdots$ plays an important role in the theory of univalent functions; for example, simple proofs of the Bieberbach conjecture for $n=4$ were arrived at through its properties [2], [3].

If $1 / f(z)=1 / z+c_{0}+c_{1} z+\cdots$ maps $|z|<1$ onto a domain $D$ such that the area (in the Lebesgue sense) of the complementary of $D$ is zero-then Grunsky's matrix is unitary [5, Theorem 1], [6, Theorem 2.2]. As Milin pointed out, the area of the complementary of $D$ is zero if and only if $\sum_{n=1}^{\infty} n\left|c_{n}\right|^{2}=1$. Following Pederson, these functions $f(z)$ will be referred as "slit mappings."

2. Properties of slit mappings. We now prove the following

TheOREM. If $f(z)=z+\sum_{n=2}^{\infty} a_{n} z^{n}$ is a slit mapping then

$$
\frac{1}{f(z)}=\frac{1}{z}+c_{0}+c_{1} z+\cdots
$$

either is of the form $1 / z+c_{0}+c_{1} z,\left|c_{1}\right|=1$, or there are infinitely many nonvanishing coefficients $c_{k}$.

Proof. The above theorem may also be formulated in the following way:

If $f(z)$ is a slit mapping such that

$$
\frac{1}{f(z)}=\frac{1}{z}+c_{0}+c_{1} z+\cdots+c_{n} z^{n}, \quad c_{n} \neq 0,
$$

then $n=1$ and $\left|c_{1}\right|=1$. 
Let

$$
P_{n}\left[\frac{1}{f(z)}\right]=F_{n}(z)=\frac{1}{z^{n}}+\sum_{k=1}^{\infty} c_{n k} z^{k}
$$

be the $n$th Faber polynomial associated with $f(z)$. Then we have by [7]

$$
c_{n k}=-n d_{n k}, \quad n, k=1,2, \cdots
$$

In terms of the coefficients $b_{n k}=(n k)^{1 / 2} d_{n k}$, we may write

$$
b_{n k}=-(k / n)^{1 / 2} c_{n k}, \quad n, k=1,2, \cdots
$$

By the unitary properties of $B$, we have

$$
\sum_{n=1}^{\infty} b_{k n} b_{j n}=0, \quad k \neq j
$$

From (4) and (5) it follows that

$$
\sum_{n=1}^{\infty} n c_{k n} \bar{c}_{j n}=0, \quad k \neq j
$$

For proof of our theorem we now assume, to the contrary, that there exist $l>1$ such that

$$
1 / f(z)=1 / z+c_{0}+c_{1} z+\cdots+c_{l} z^{l},
$$

where $c_{l} \neq 0$.

Subtitution of $k=1, j=l^{2}$ in (6) yields

$$
\sum_{n=1}^{\infty} n c_{1 n} \bar{c}_{l^{2}, n}=0 \text {. }
$$

Since

$$
P_{1}\left[\frac{1}{f(z)}\right]=\frac{1}{z}+\sum_{k=1}^{\infty} c_{1 k} z^{k}=\frac{1}{f(z)}-c_{0}=\frac{1}{z}+\sum_{k=1}^{l} c_{k} z^{k}
$$

it follows that

$$
c_{1 k}=c_{k}, \quad k=1,2, \cdots, l, \quad c_{1 k}=0 \text { for } k>l .
$$

From (8) and (9) we obtain

$$
\sum_{n=1}^{l} n c_{1 n} \bar{c} t^{2}, n=0
$$


Since $P_{n}(x)$ is a polynomial of degree $n$ in $x$, we have by (7), for any natural $n$,

$$
\begin{gathered}
P_{n}\left[\frac{1}{f(z)}\right]=\frac{1}{z^{n}}+\sum_{k=1}^{l n} c_{n k} z^{k} \\
c_{n, l n}=\left(c_{1 l}\right)^{n}=\left(c_{l}\right)^{n}, c_{n k}=0 \quad \text { for } k>\ln .
\end{gathered}
$$

From the definition of the coefficients $d_{n k}$, it is clear that $d_{n k}=d_{k n}$. Following Schiffer we deduce from (3)

$$
k c_{n k}=n c_{k n}, \quad n, k=1,2, \cdots .
$$

(This identity was first proved by Grunsky [4] and Schur [8].) From (11) and (12), we have

$$
c_{k n}=0, \quad k>\ln .
$$

Substituting $k=l^{2}$ in (13) we get

$$
c_{l^{2}, n}=0, \quad n=1,2, \cdots, l-1 .
$$

Using (11) for $n=l$ and (12) for $k=l^{2}, n=l$ it follows that

$$
l^{2} c_{l, l^{2}}=l c_{l^{2}, l}=l^{2}\left(c_{l}\right)^{l}=l^{2}\left(c_{1 l}\right)^{l}
$$

equations (14) and (15) now yield

$$
\sum_{n=1}^{l} n c_{1 n} \bar{c}_{l^{2}, n}=l^{2} c_{1 l}\left(\bar{c}_{1 l}\right)^{l}=l^{2} c_{l}\left(\bar{c}_{l}\right)^{l} \neq 0 .
$$

Since this contradicts $\left(8^{\prime}\right)$ we have proved that if $f(z)$ is a slit mapping such that

$$
\frac{1}{f(z)}=\frac{1}{z}+c_{0}+c_{1} z+\cdots+c_{n} z^{n}, \quad c_{n} \neq 0
$$

then necessarily $n=1$. But it follows then, from the condition $\sum_{k=1}^{\infty} k\left|c_{k}\right|^{2}=1$, that $\left|c_{1}\right|=1$, and the proof is complete.

REMARK 1. In [1] the author considered properties of slit mappings and proved the above theorem for some particular cases.

REMARK 2. The above theorem contains a result of Pederson [6, Theorem 2.3], as a special case.

\section{REFERENCES}

1. D. Aharonov, The Schwarzian derivative and univalent functions, Thesis, Technion-Israel Institute of Technology, 1967.

2. A. Charzynski and M. Schiffer, A new proof of the Bieberbach conjecture for the fourth coefficient, Arch. Rational Mech. Anal. 5 (1960), 187-193. 
3. P. R. Garabedian, G. G. Ross and M. Schiffer, On the Bieberbach conjecture for even $n$, J. Math. Mech. 14 (1965), 975-988.

4. H. Grunsky, Koeffizientenbedingungen fïr Scklicht abbildende meromorphe Funktionen, Math. Z. 45 (1939), 29-61.

5. I. M. Milin, The area method in the theory of univalent functions, Dokl. Akad. Nauk SSSR 154 (1964), 264-267=Soviet Math. Dokl. 5 (1964), 78-81.

6. R. N. Pederson, On unitary properties of Grunsky's matrix, Arch. Rational Mech. Anal. 29 (1968), 370-377.

7. M. Schiffer Faber polynomials in the theory of univalent functions, Bull. Amer. Math. Soc. 54 (1948), 503-517.

8. I. Schur, On faber polynomials, Amer. J. Math. 67 (1945), 33-41.

Israel Institute of Technology, Technion, Haifa, Israel 